\title{
Biomodifications of resveratrol by Phanerochaete chrysosporium
}

\author{
MAEgoRZATA BRZEZIŃSKA-RODAK *, KATARZYNA LUBIAK, MAGDALENA KLIMEK-OCHAB, \\ Paulina MajewsKa, EWA ŻYMańcZYK-DUdA \\ Wrocław University of Science and Technology, Wrocław, Poland
}

\begin{abstract}
Compounds of plant origin showing antioxidative activity are important due to their ability to decrease oxidative stress. They also protect against the harmful effects of the free radicals formed as by-products of some metabolic pathways or as external factors. This supports the application of these compounds (e.g., vitamin C) as additives of food, pharmaceuticals (vitamin E, curcumin, etc.), and cosmetics (e.g., ellagic acid). Natural antioxidants are mainly isolated from plant sources, but besides these, novel, effective, and "green" methods of obtaining antioxidative compounds are being investigated. One of the natural antioxidants is resveratrol belonging to the stilbene polyphenol family. It occurs naturally in grape, and is produced by cells in response to fungal infections or a potential stress factor (e.g., tissue damage). The beneficial effect of resveratrol is based on the inhibition of lipid oxidation and cardioprotective properties. The biocatalyzed synthesis of $O$-methylated derivatives of resveratrol is especially interesting from the point of view of their possible applications as anticancer agents. The fungal species Phanerochaete chrysosporium is known for its ability to produce methyltransferases, and therefore used as a biocatalyst in resveratrol modifications. Increased production of methyltransferase was stimulated in the strain by changing the conditions of both its cultivation and biotransformation. However, such an approach resulted in the conversion of resveratrol into 3,5-dihydroxybenzaldehyde, indicating a partial degradation of the substrate.
\end{abstract}

Key words: resveratrol, biotransformation, white-rot fungi

\section{Introduction}

Natural phenolic antioxidants of plant origin have been investigated for many years. The research conducted so far has focused on the evaluation of their biological activity (Kris-Etherton et al., 2002; Montedoro et al., 1992; Manna et al., 1999; Visioli et al., 1998a, 1998b; Adom et al., 2003; Gallardo et al., 2006) and discovering effective pathways for the synthesis of compounds with antioxidative activity or their modification to produce molecules with interesting biological properties (McGuire et al., 1999; Barluenga et al., 2006; Fillion et al., 2006). Phenolic antioxidants also act as antimicrobial, anti-inflammatory, and anticancer agents (Roberti et al., 2003; Baur and Sinclair, 2006; Houille et al., 2014). Current scientific reports indicate that the presence of one of the natural phenolic antioxidants - resveratrol in the common diet significantly decreases the risk of heart diseases and the aging of cells related to the presence of the free radicals and decrease in the natural defense mechanisms of the body (Catalgol et al., 20012; Stervbo et al., 2007). Due to these health benefits of resveratrol, there is an urgent need to find novel methods for the synthesis of this polyphenol and its derivatives. Especially, the biocatalyzed synthesis of $O$-methylated derivatives of resveratrol is interesting because of their potential applications as anticancer agents. A trimethyl derivative of resveratrol (3,5, $4^{\prime}$-trimethoxystilbenoid) inhibits the mobility of abnormal hepatocytes that have become cancerous (Dipak and Maulik, 2006) and shows cytotoxic effects on the cancer cells of the large intestine

\footnotetext{
* Corresponding author: Wrocław University of Science and Technology, Wrocław, Poland; e-mail: malgorzata.brzezinska-rodak@pwr.edu.pl
} 
(Mazue et al., 2010). In addition, the introduction of methyl groups increases the bioaffinity of a resveratrol derivative, which assures better access of the compound into the living cells by improving their lipophilic properties (Jeandet et al., 2014). Methylation also improves the stability of resveratrol under physiological conditions, simply by protecting the molecule from detoxification and aiding in the excretion of the xenobiotics from the organism. Resveratrol and its derivatives are currently being obtained from natural sources (Japanese knotweed, grape skins) (Jeandet et al., 2014; Wanga et al., 2013), but the methods used are inefficient and expensive (Wang et al., 2008; Zhuang et al., 2008) due to the low concentration of the desired compounds in the raw materials. This necessitates the development of alternative chemical and biocatalytic strategies for the synthesis and modifications of resveratrol. The chemical synthesis of resveratrol is carried out by the Perkin reaction (Solladie et al., 2003), Wittig reaction, Horner -Wadsworth-Emmons reaction (Yu et al., 2002), or the Heck reaction (Botella and Najera, 2004), which requires the use of expensive and often toxic reagents. As in the case of bioconversion, genetically modified microorganisms or plant cell cultures are employed in the synthesis of resveratrol (Jeandet et al., 2014; Horinouchi, 2009; Kang et al., 2014; Rimando et al., 2012). Mono-, di-, and trimethylated derivatives of resveratrol were obtained in a study using the modified cells of Escherichia coli, but the scale of production was small, resulting in a low yield in the range of milligrams (Kang et al., 2014). Therefore, the development of effective synthetic methods for obtaining and modifying resveratrol and its derivatives may be a valuable solution to this important and contemporary issue, especially considering the possibility of synthesizing novel derivatives possessing important biological properties.

In the past several decades, many biocatalytic processes have been developed as useful alternatives to the traditional chemical methods of synthesis (Wachtmeister and Rother, 2016). The application of isolated enzymes or whole-cell biocatalysts allows a simple and cost-effective synthesis of the desired compounds. Additionally, because of their low cost and higher stability, whole-cell biocatalysts are often the preferred tools for biocatalysis (Straathof, 2013; de Carvalho, 2017).

Fungi are recognized for their superior ability to produce a large variety of enzymes and metabolites that can be used for different applications. Among them, a whiterot fungus, Phanerochaete chrysosporium, has been the subject of intensive research, related mostly to the degradation of a wide range of recalcitrant xenobiotic compounds and mineralization of persistent aromatic pollutants (Chagas and Durrant, 2001; Gao et al., 2010; Yu et al., 2006; Zhang et al., 2008). Coulter et al. (1993) succeeded in the isolation and characterization of an $S$-adenosyl methionine (SAM)-dependent, 2,4-disubstituted phenol $O$-methyltransferase from $P$. chrysosporium. The available literature has limited data on the nature and function of $O$-methyltransferase in white-rot fungi, but a study suggested that methylation is applied to remove the free hydroxyl phenolic compounds derived from the degradation of lignin, which are known to be toxic to the fungal cells (Thanh Mai Pham and Kim, 2016). Thus, the present study aims at modifying resveratrol by an $O$-methylation process using the mycelium of $P$. chrysosporium under an effective biotransformation condition.

\section{Materials and methods}

\section{Microorganisms and culture conditions}

The fungal strain $P$. chrysosporium CCM 8024 was purchased from the Czech Collection of Microorganisms and cultured on Petri dishes with potato dextrose agar (PDA) at $4{ }^{\circ} \mathrm{C}$. Biomass cultivation was initiated by transferring three excision fragments $(1 \mathrm{~cm} \times 1 \mathrm{~cm}$ square $)$ of mycelium cultivated on a solid medium (PDA) to $100 \mathrm{ml}$ of a liquid potato dextrose broth (PDB) or a modified mineral medium $\left(\mathrm{g} / \mathrm{dm}^{3}: \mathrm{KH}_{2} \mathrm{PO}_{4}-0.2, \mathrm{MgSO}_{4} \cdot 7 \mathrm{H}_{2} \mathrm{O}\right.$ $-0.05, \mathrm{CaCl}_{2} \cdot 2 \mathrm{H}_{2} \mathrm{O}-0.013$, yeast extract -0.1 , glycerol - 10, L-asparagine - 1.13, $\mathrm{NH}_{4} \mathrm{NO}_{3}$ - 0.5, 2,2-dimethylsuccinic acid - 1.46, $\mathrm{NH}_{4} \mathrm{H}_{2} \mathrm{PO}_{4}-2.0, \mathrm{~K}_{2} \mathrm{HPO}_{4}-0.4$; $\mathrm{mg} / \mathrm{dm}^{3}$ : ferric citrate $-12, \mathrm{ZnSO}_{4} \cdot 7 \mathrm{H}_{2} \mathrm{O}-6.6$, $\mathrm{MnSO}_{4} \cdot \mathrm{H}_{2} \mathrm{O}-5.0, \mathrm{CoCl}_{2} \cdot 6 \mathrm{H}_{2} \mathrm{O}-1.0, \mathrm{CuSO}_{4}-1.0$, thiamine - 0.1; $\mathrm{pH} 5.5$; glycerol was added after sterilization). This was followed by incubation under shaking (120 rpm) for 5 (on PDB) or 14 days (on mineral medium) at $26^{\circ} \mathrm{C}$. (For the biotransformation of resveratrol, the biomass cultivated on the modified mineral medium (described above) under stationary conditions $\left(26^{\circ} \mathrm{C}\right.$ for 14 days) was used.) After incubation, the biomass was separated from the medium by centrifugation (10 min, $4000 \mathrm{rpm}, 4^{\circ} \mathrm{C}$ ), washed with sterile water, and centrifuged again under the same conditions. 


\section{Chemicals}

Resveratrol and its trimethyl derivative were purchased from TCI (Tokyo Chemical Industry). PDB and agar agar were obtained from Biocorp. Acetonitrile of highperformance liquid chromatography (HPLC) grade was purchased from Merck (Darmstadt, Germany). Thiamine, 2,2-dimethylsuccinic acid (analytical grade), SAM, and ferric citrate were purchased from Sigma-Aldrich (formally Merck). Ethyl acetate (analytical grade), acetic acid, hexane, and the rest of the chemicals were purchased from POCH (Polish Chemicals Reagents).

\section{Biotransformation procedures}

For biotransformation processes, $5 \mathrm{~g}$ of the washed biomass was added to $100 \mathrm{ml}$ of water (in $250 \mathrm{ml}$ Erlenmeyer flask) and used. The processes were carried out according to the following protocols:

Protocol 1. After cultivation on PDB, the biomass was transferred to $100 \mathrm{ml}$ of water containing $30 \mathrm{mg}$ of resveratrol. Biotransformation was carried out under shaking $(120 \mathrm{rpm})$ for 7 days at $26^{\circ} \mathrm{C}$. The progress of the reaction was monitored at 24 -h intervals.

Protocol 2. After cultivation on the liquid potato medium, the biomass was transferred to $100 \mathrm{ml}$ of water containing $30 \mathrm{mg}$ of wood sawdust and $30 \mathrm{mg}$ of resveratrol. Biotransformation was carried out under shaking $(120 \mathrm{rpm})$ for 7 days at $26^{\circ} \mathrm{C}$. The progress of the reaction was monitored at 24 -h intervals.

Protocol 3. After cultivation on the liquid potato medium, the biomass was transferred to $100 \mathrm{ml}$ of water containing $30 \mathrm{mg}$ of wood sawdust and incubated at $26^{\circ} \mathrm{C}$ for $24 \mathrm{~h}$ on a rotary shaker (120 rpm). After incubation, $30 \mathrm{mg}$ of resveratrol was added and biotransformation was carried out under the same conditions for the next 7 days. The progress of the reaction was monitored at 24 -h intervals.

Protocol 4. The biomass cultivated on the modified mineral medium under stationary conditions (without shaking, at room temperature (RT)) was transferred to an Erlenmeyer flask containing $100 \mathrm{ml}$ of distilled water and one of the following ingredients:
a) $30 \mathrm{mg}$ of resveratrol,
b) $30 \mathrm{mg}$ of resveratrol and $15 \mathrm{mg}$ of $L$-methionine,
c) $50 \mu \mathrm{l}$ of $(1 \mathrm{mM}) \mathrm{SAM}$ and $30 \mathrm{mg}$ of resveratrol,
d) $100 \mu \mathrm{l}$ of $(1 \mathrm{mM}) \mathrm{SAM}$ and $30 \mathrm{mg}$ of resveratrol.

All the flasks were incubated without shaking at RT for 7 days. The progress of the reaction was monitored at 24 -h intervals.
Protocol 5. Biotransformations were carried out under the same conditions described for Protocol 4, but in these set of experiments, the biomass was added after the 24-h incubation in the water-starvation step.

Protocol 6. The biomass cultivated on the modified mineral medium under shaking (120 rpm) was transferred to an Erlenmeyer flask containing $100 \mathrm{ml}$ of distilled water, $10 \mathrm{mg}$ of hydroconiferyl alcohol, and $30 \mathrm{mg}$ of resveratrol. The biotransformation was carried out for 14 days at $26^{\circ} \mathrm{C}$ under shaking (120 rpm). The progress of the reaction was monitored at 24 -h intervals.

Protocol 7. The biomass cultivated on the mineral medium was incubated in $100 \mathrm{ml}$ of water with $10 \mathrm{mg}$ of hydroconiferyl alcohol for $24 \mathrm{~h}$. After incubation, the biotransformation medium was supplemented with one of the following:

a) $30 \mathrm{mg}$ of resveratrol,

b) $30 \mathrm{mg}$ of resveratrol and $200 \mu \mathrm{l}$ of $(1 \mathrm{mM}) \mathrm{SAM}$,

c) $30 \mathrm{mg}$ of resveratrol and $15 \mathrm{mg}$ of $L$-methionine,

d) $30 \mathrm{mg}$ of resveratrol, $200 \mu \mathrm{l}$ of $(1 \mathrm{mM}) \mathrm{SAM}$, and $15 \mathrm{mg}$ of $L$-methionine.

The biotransformation was carried out for 14 days at $26^{\circ} \mathrm{C}$ under shaking (120 rpm). The progress of the reaction was monitored at 24 -h intervals. All the experiments were done in triplicate.

\section{Biotransformation in semi-preparative scale}

The thin-layer chromatography (TLC) analysis allowed selecting the optimal conditions for the biotransformation process which were found to be met by Protocol $7 \mathrm{~b}$. The biotransformation was carried out in Erlenmeyer flasks for 2 and 6 days (ten flasks for each set of experiments), and then the mixture of the products was isolated as described below.

\section{Isolation and preliminary analysis of the products of biotransformation}

After biotransformation, the biomass was separated by gravity filtration and the biotransformation medium was extracted twice with ethyl acetate. Following extraction, the organic solvent was dried under anhydrous magnesium sulfate and evaporated. All the samples were first analyzed by TLC using silica gel plates with a fluorescent indicator, $\mathrm{F}_{254}$. The chromatogram was developed using an eluent (hexane: ethyl acetate: acetic acid, $5: 4: 1, \mathrm{v} / \mathrm{v} / \mathrm{v})$ and detected using an ultraviolet lamp $(\lambda=240 \mathrm{~nm})$. 


\section{HPLC analysis of the selected samples}

After isolation and TLC, the selected samples were analyzed with an HPLC system (Gold Nuveaux, Beckman). The products were detected (at $\lambda=285 \mathrm{~nm}$ ) using a Supelcosil LC-18 HPLC Column ( $5 \mu \mathrm{m}$ particle size, $25 \mathrm{~cm} \times 4.6 \mathrm{~mm}$ ) with mobile phases A (water:acetic acid, 99:1, v/v) and B (acetonitrile). The elution gradient was as follows: from 10 to $35 \% \mathrm{~B}$ for $15 \mathrm{~min}$, from 35 to $90 \%$ B for $5 \mathrm{~min}, 90 \%$ B for $3 \mathrm{~min}$, from 90 to $10 \%$ B for $1 \mathrm{~min}$, and $10 \% \mathrm{~B}$ for $16 \mathrm{~min}$. The flow rate was $1 \mathrm{ml} / \mathrm{min}$. The injection volume was $20 \mu \mathrm{l}$. The column temperature was set at $28^{\circ} \mathrm{C}$.

\section{Separation and analysis of 3,5-dihydroxybenzaldehyde}

After the HPLC analysis, the best biotransformation conditions were applied on a semi-preparative scale. The products were separated by a medium-pressure liquid chromatography system (Interchim PuriFlash 430evo) on a reversed-phase column (120 g, PuriFlash C18-HP) with a grain size of $15 \mu \mathrm{m}$.

The applied elution gradient was as follows: $4 \mathrm{~min}$ of isocratic flow of pure water, $3 \mathrm{~min}$ from 0 to $5 \%$ of acetonitrile in water, $5 \mathrm{~min}$ of isocratic flow of $5 \%$ of acetonitrile in water, $5 \mathrm{~min}$ from 5 to $10 \%$ of acetonitrile in water, $5 \mathrm{~min}$ of isocratic flow of $10 \%$ of acetonitrile in water, $3 \mathrm{~min}$ from 10 to $15 \%$ of acetonitrile in water, 6 min of isocratic flow of $15 \%$ of acetonitrile in water, $5 \mathrm{~min}$ from 15 to $20 \%$ of acetonitrile in water, $8 \mathrm{~min}$ of isocratic flow of $20 \%$ of acetonitrile in water, $17 \mathrm{~min}$ from 20 to $100 \%$ of acetonitrile in water, and $45 \mathrm{~min}$ of isocratic flow of pure acetonitrile. The purified fractions containing the product were collected, evaporated, and analyzed by nuclear magnetic resonance (NMR) (Bruker Avance $^{\mathrm{TM}} 600 \mathrm{MHz}$ ) and mass spectroscopy (Waters GCT Premier). The structure was determined as 3,5-dihydroxybenzaldehyde: ${ }^{1} \mathrm{H}$ NMR (DMSO- $d_{6}, \delta$, ppm): 6.51 (t, $J=2.3 \mathrm{~Hz}, 1 \mathrm{H}, \mathrm{C} \underline{\mathrm{H}}-\mathrm{C} 4), 6.73(\mathrm{~d}, J=2.3 \mathrm{~Hz}, 2 \mathrm{H}, \mathrm{C} \underline{\mathrm{H}}-$ C2, C $\underline{H}-\mathrm{C} 6), 9.78$ (s, 1H, C $\underline{H} \mathrm{O}) ;{ }^{13} \mathrm{C}$ NMR, ${ }^{13} \mathrm{C}-{ }^{1} \mathrm{H}$ HSQC NMR, ${ }^{13} \mathbf{C}-{ }^{1} \mathbf{H}$ HMBC NMR (DMSO- $d_{6}, \delta$, ppm): 107.33 (2C, ㄸH-C2, $\underline{\mathrm{C}} \mathrm{H}-\mathrm{C} 6), 108.68$ (1C, $\underline{\mathrm{C} H}-\mathrm{C} 4), 138.33$ (1C, CCHO-C1), 159.13 (2C, $\underline{\mathrm{COH}}-\mathrm{C} 3, \underline{\mathrm{COH}}-\mathrm{C} 5), 193.16$ (1C, CHO). MS (TOF MS ES+) calcd. for $\mathrm{C}_{7} \mathrm{H}_{7} \mathrm{O}_{3}[\mathrm{M}+\mathrm{H}]^{+}$: 139.0395; found: 139.0758 .

\section{Results and discussion}

Bioconversion with living microbial cells exhibiting a wide range of enzymatic activities is a useful tool to carry out bioorganic synthesis. One of the most important advantages of microbial biotransformations is the possibility to activate or induce the desired enzymatic activities simply by applying suitable preincubation conditions. These include temperature pretreatment, cultivation under anaerobic environment or starvation (Paszczyński and Łobarzewski, 1984; Jayashree et al., 2014), and the use of different types of cultivation media with different sources of supporting elements, or additives of special purposes (e.g., activators, inhibitors, donors of hydrogen, methyl, or sulfur groups) (Żymańczyk-Duda et al., 2015; Kozyra et al., 2013; Coque et al., 2003).

The production of bioactive compounds in fungi usually occurs during the stationary phase of growth, often as a response to stress factors. Parameters such as the type or concentration of nutrients, incubation times, $\mathrm{pH}$, temperature, and others that influence the production of desired compounds can be easily modified (Demain, 2000).

The present study focused on obtaining the methyl derivatives of resveratrol using the fungal species P. chrysosporium. Despite the difficulties in cultivation, this microorganism was chosen because of its natural ability to produce methyltransferases at the early stage of the degradation of lignin to methylate phenolic compounds, which finally inhibit the activity of lignin peroxidase (Than Mai Pham and Kim, 2016). Thus, it seemed possible to stimulate the microbial cells to achieve increased production of this type of enzymes through simple medium engineering to induce the methylation of resveratrol (Coque et al., 2003). Biotransformations were carried out according to different protocols (described in the "Materials and methods" section). Various culture media were used (PDB, modified mineral medium), in addition to two methyl donors (SAM, $L$-methionine) that were used in three combinations and special additives such as sawdust and hydroconiferyl alcohol. In the experiments carried out according to Protocol 1, the fungi were cultured on a potato medium, so that the cells had constant access to an easily absorbable carbon (dextrose) source. As a result, they were unable to use the exogenous substrate as a source of carbon and energy, and therefore, no resveratrol modifications were observed. Hence, the TLC analysis indicated the presence (data not shown) of the tested substrate (resveratrol) after each day of biotransformation (Protocol 1).

On the basis of the abovementioned observations, and the knowledge about the natural abilities of the stu- 


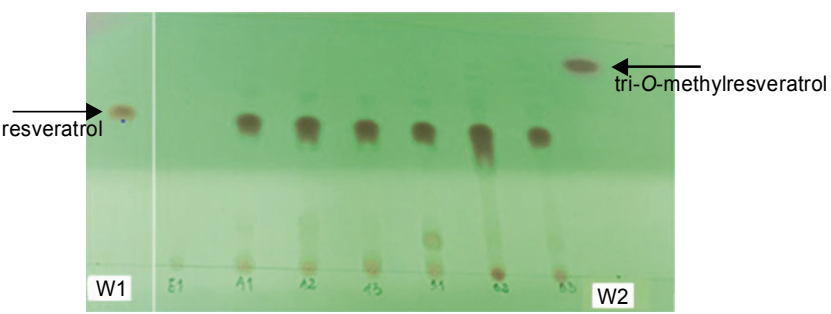

Fig. 1. Biotransformation of resveratrol with the addition of wood sawdust. A1-A3 - Protocol 2; B1-B3 - Protocol 3; E1 - biotransformation without sawdust; W1 - standard of resveratrol; W2 - standard of tri- $O$-methylresveratrol

died microorganism to degrade lignin (Thanh Mai Pham and Kim, 2016), wooden sawdust (natural substrate) was added to the biotransformation medium. The experiments were carried out according to Protocols 2 and 3. Control experiments without the addition of sawdust were also performed. As can be seen in Figure 1, in the control sample, resveratrol was completely degraded and utilized by the cells. In contrast, in the experimental sample containing wood sawdust, resveratrol was slightly modified (found by a small number of transformation products), indicating that in the tested conditions the degraded lignin served as a source of carbon and energy. A few products of resveratrol transformation (signals other than that of resveratrol) can be observed in Figure 1, which may indicate the low activity of methyltransferases and the formation of methyl derivatives of the substrate (signals with a similar retention factor (Rf) as tri- $O$-methylresveratrol standard- samples B2 and B3). Unfortunately, the efficiency of discussed processes was very low and it was impossible to isolate and determine the structures of the mentioned derivatives.

The next set of experiments involved the cultivation of biomass on the modified mineral medium, selected to meet the requirements of nutrition and $\mathrm{pH}$ of the tested strain. In the experiments carried out according to Protocol 4a, the biotransformation time was extended to 10 days to check the ability of the biocatalyst cells to transform resveratrol after cultivation under limited access to nutrients. Unfortunately, no formation of tri- $O$-methyl derivatives was observed, and only a slow degradation of the substrate was noted (days 10-14, data not shown). Probably, the cultivation medium used provided sufficient amounts of nutrients and only after exhausting the stored nutrients, the cells were forced to partially mineralize resveratrol to use this compound as

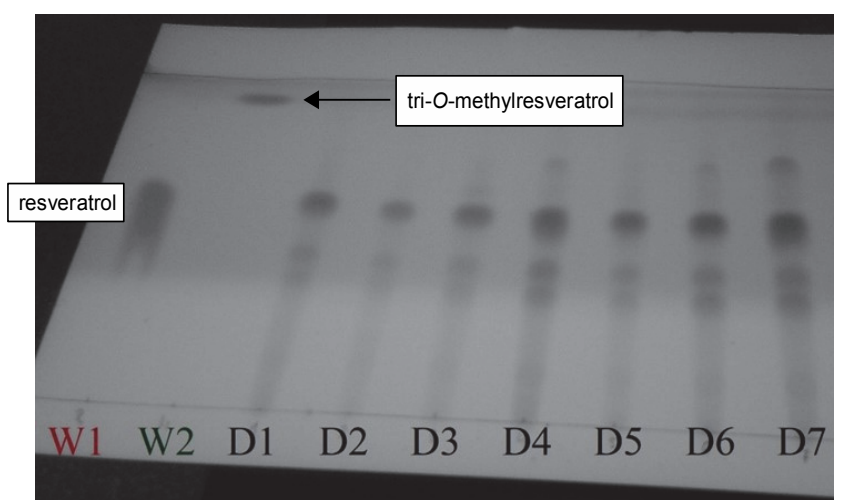

Fig. 2. TLC analysis of the biotransformations carried out according to Protocol 7a. W1 - standard of resveratrol; $\mathrm{W} 2$ - standard of tri- $O$-methyl derivative of resveratrol; D1-D7 - samples after biotransformations

a source of carbon and energy. It is known from the literature that the synthesis of enzymes by $P$. chrysosporium is strongly affected by the presence of $\mathrm{O}_{2}$ (Dosoretez et al., 1990). For example, the production of extracellular proteases and polysaccharides is high under continuous oxygenation and correlated with a fast decay of lignin (Dosoretez et al., 1990). Thus, to avoid the degradation of resveratrol, the biomass obtained by cultivation under stationary conditions with limited access to oxygen was applied in Protocol 5. However, the biomass showed no enzymatic activity towards resveratrol (data not shown), probably because the oxygen-restricted conditions caused the tested strain to follow a different pattern of growth and exhibit a different profile of enzymatic activities (Somerville and Proctor, 2013).

To simulate the synthesis of methyltransferases, hydroconiferyl alcohol, a compound occurring naturally during the degradation of lignin by $P$. chrysosporium, was used as an additive in the biotransformation medium. Hydroconiferyl alcohol, sinapyl alcohol, and coumaryl alcohol are the components of the cell wall of plants (Koutaniemi et al., 2005). During the process of initial delignification, these compounds are released, and because they are toxic to the cells, they undergo $O$-methylation (Thanh Mai Pham and Kim, 2016). The idea behind the next set of experiments was the introduction of stress conditions by adding alcohol (hydroconiferyl alcohol) to force an increased synthesis of methyltransferases which are crucial in neutralizing the toxic substances. Simultaneously, resveratrol was added to the cultivation medium and, assuming that the methyltransfera- 


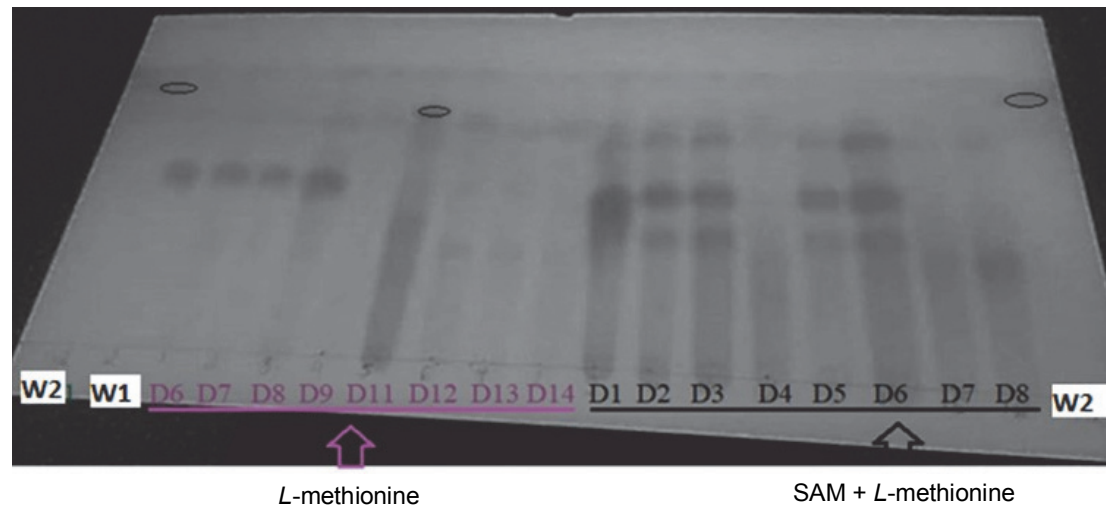

Fig. 3. Biotransformation of resveratrol with the addition of $L$-methionine (Protocol 7c: days 6-14) and SAM and $L$-methionine (Protocol 7d: days 1-8). W1 - standard of resveratrol; $\mathrm{W} 2$ - standard of tri- $O$-methylresveratrol (marked with circles)

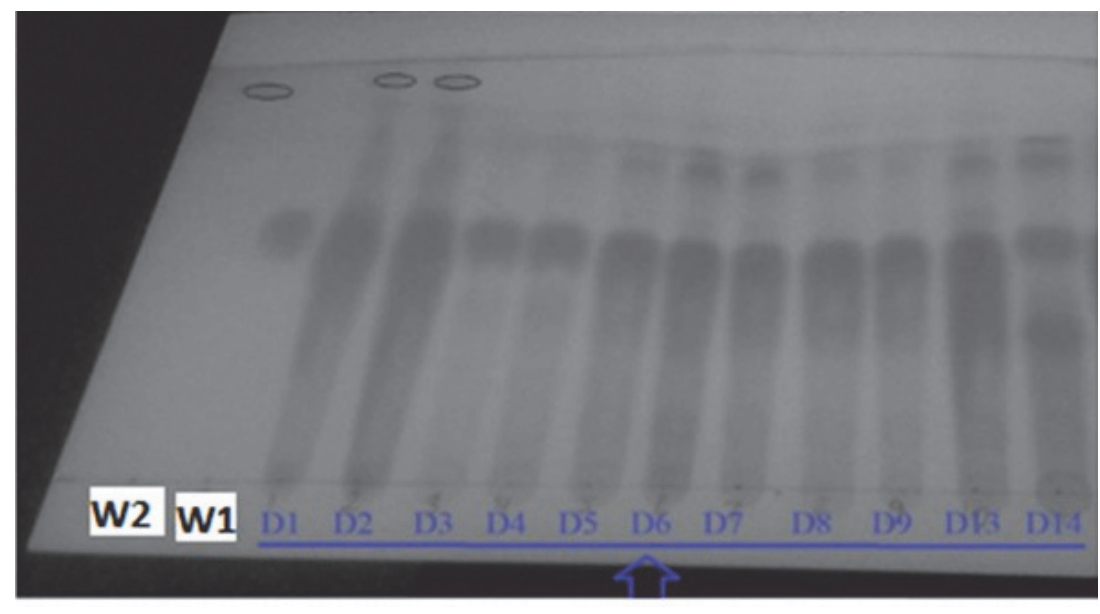

S-adenosylmethionine

Fig. 4. Biotransformation of resveratrol with SAM $(200 \mu \mathrm{l})$ as an additive (Protocol 7b). W2 - standard of tri- $O$-methylresveratrol (marked with a circle); W1 standard of resveratrol. Probably methylated derivative of resveratrol (D1 and D2 sample) is marked with a circle

ses produced by the fungal strain have a low substrate specificity, methylation of the substrate was expected. However, the simultaneous use of resveratrol and the addition of hydroconiferyl alcohol promoted degradation and gradual mineralization of the main substrate (resveratrol). Therefore, in the next set of experiments, the addition of the substrate followed (after $24 \mathrm{~h}$ ) the addition of hydroconiferyl alcohol. As shown in Figure 2, resveratrol underwent a partial conversion to several products (signals for samples D1-D7) that could be the result of both degradation processes (signals with $\mathrm{Rf}$ lower than that of the substrate - W1) and other enzymatic activities (samples D1-D7; Rf higher than that of the substrate standard W1). Apparently, the presence of hydroconiferyl alcohol, which was toxic to the cells, during the biomass preincubation induced the synthesis of an enzyme neutralizing this harmful factor. Perhaps, this enzyme (4-O-methyltransferase) (Thanh Mai Pham and Kim, 2016) was able to transfer the methyl groups on the 4-OH position in the aromatic ring of hydroconiferyl alcohol, and as a consequence, the presence of a derivative with a signal between the substrate standard and the target product ( $O$-methyl derivative of resveratrol) was observed (Fig. 2, line D7).

Therefore, to stimulate the synthesis of methyltransferases further, a 24-h starvation period was introduced (incubation in water after cultivation-Protocol 5), methyl donors were added ( $L$-methionine and/or SAM - Proto 


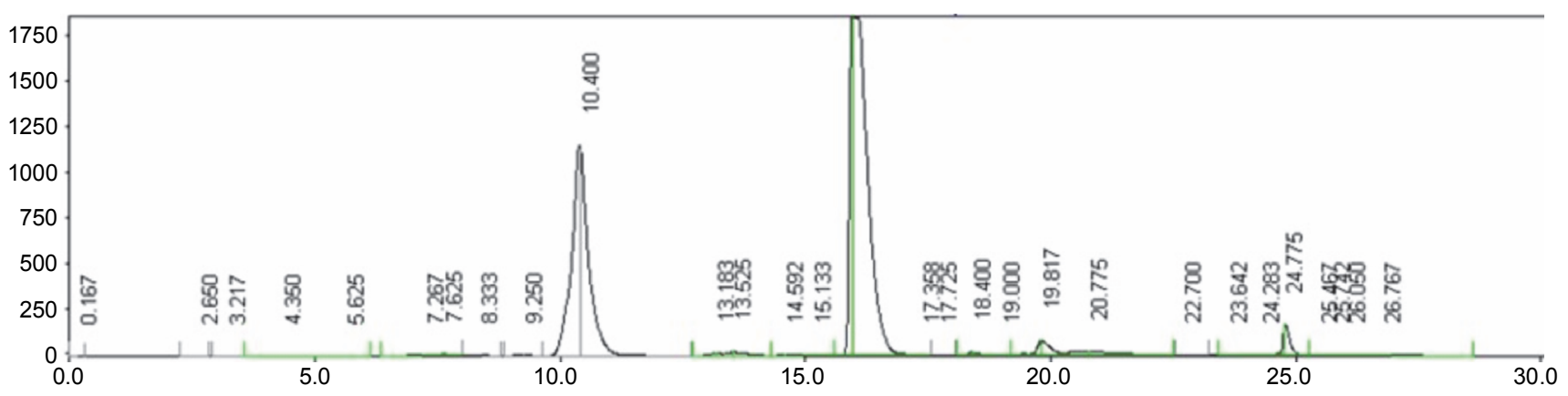

Fig. 5. HPLC analysis of a sample obtained 2 days after the biotransformation of resveratrol (Protocol $7 \mathrm{~b}$ ). Retention time: resveratrol - $16 \mathrm{~min}$; 3,5-dihydroxybenzaldehyde - $10.40 \mathrm{~min}$; unidentified derivative $-24.77 \mathrm{~min}$

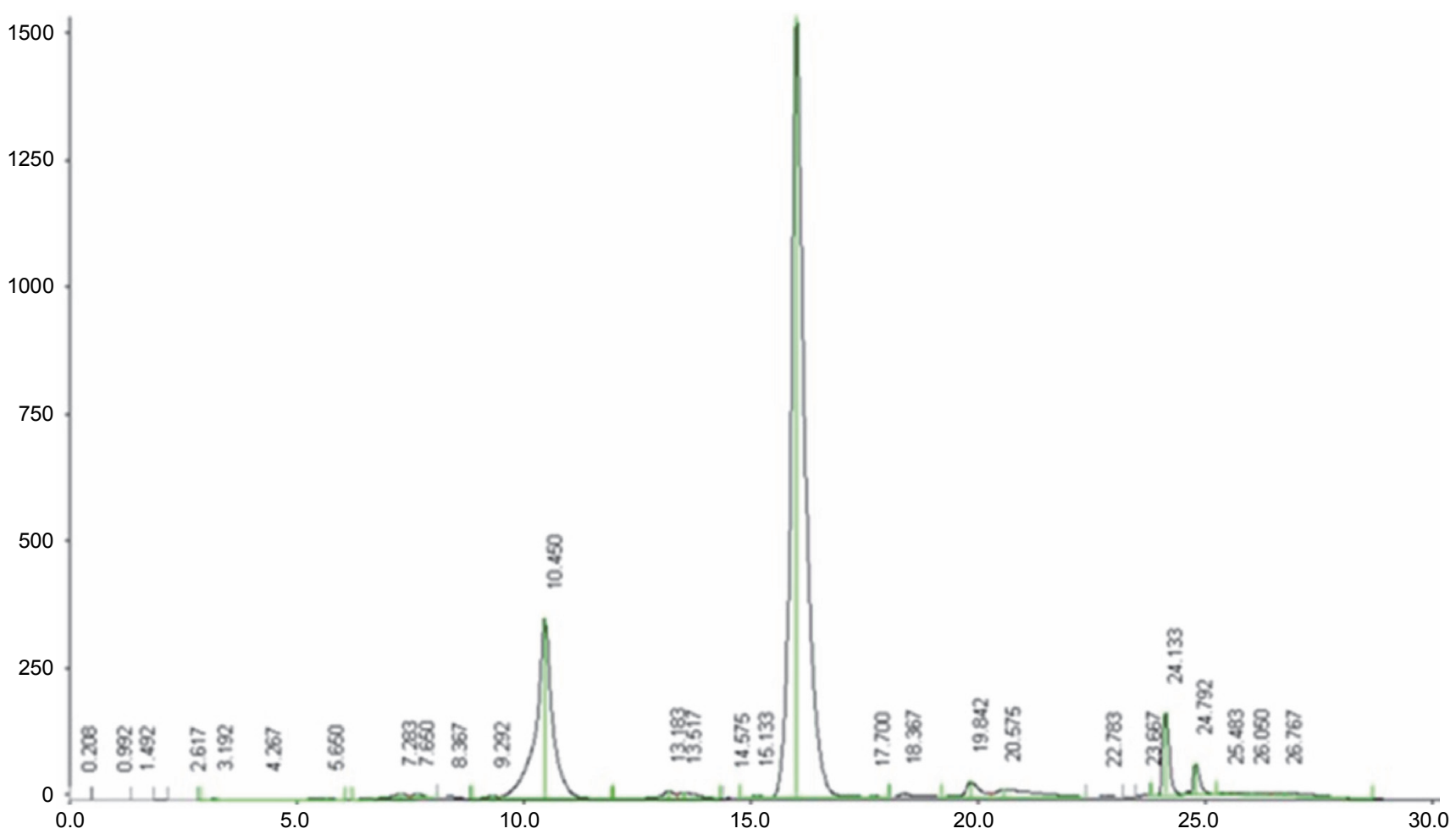

Fig. 6. HPLC analysis of a sample obtained 2 days after the biotransformation of resveratrol. HPLC analysis performed after the addition of a standard of substrate (retention time $=16 \mathrm{~min}$ ) and tri- $O$-methyl derivative standard (retention time $=24.13 \mathrm{~min}$ ) to the analyzed sample

cols $4 b-d, 5 b-d, 7 b-d)$ to the biotransformation medium, and the incubation time for biotransformation was extended to 14 days. SAM has been previously applied as a methyl donor in the methylation of the 4-hydroxy groups in 3-methoxy- and 3,5-dimethoxy-substituted 4-hydroxybenzaldehydes, 4-hydroxybenzoic acids, and 4-hydroxyacetophenones (Coulter et al., 1993), and appears to be responsible for the 4-O-methylation of the degradation products of lignin (Chen et al., 1982; Eriksson et al., 1984; Jeffers et al., 1997). There is a chance that the same enzyme was involved in the methylation of resveratrol (2,4-substituted phenol $O$-methyltransferase; Coulter et al., 1993). To check this possibility, in the subsequent experiments, $L$-methionine was introduced into the reaction medium. In the experiments performed according to Protocols $4 \mathrm{~b}, 5 \mathrm{~b}$, and $7 \mathrm{c}$, methionine was added simultaneously with resveratrol because it is known that the physiological donor of methyl groups (SAM) is formed in the cells from $L$-methionine and ATP (Kim et al., 2003). In the experiments performed accor- 
ding to Protocols $4 \mathrm{~b}$ and $5 \mathrm{~b}$, no positive effects on the methyltransferase activity were observed. The natural amino acid ( $L$-methionine) added to the medium in these experiments was utilized by the cells (as a source of energy and nitrogen). However, when analyzing the experiments performed according to Protocol 7c (Fig. 3, samples D6-D8 for $L$-methionine part in TLC analysis), a constant concentration of resveratrol was noted in the tested samples until the eighth day of the bioconversion process, while in the following days the substrate was transformed to small amounts of less polar derivatives (Fig. 3, samples D11-D14). One of these products (D11signal marked with a circle) had an Rf of 0.811 similar to the tri- $O$-methyl derivative standard having an $\mathrm{Rf}$ of 0.89 . It is therefore possible that this was a derivative of resveratrol with one or two methoxyl groups in the structure. Unfortunately, again, a small sample made it impossible to determine the structure of the product.

To improve the efficiency of transformation of resveratrol, in the next stage of research, different concentrations (50,100, and $200 \mu \mathrm{l}$ of $1 \mathrm{mM}$ solution - Protocols $4 \mathrm{c}, 4 \mathrm{~d}, 5 \mathrm{c}, 5 \mathrm{~d}$, and $7 \mathrm{~b}$ ) of SAM were tested to determine the sufficient amount of this compound that would serve to methylate resveratrol. As shown in Figure 4 , after 1 and 2 days of biotransformation with the addition of $200 \mu \mathrm{l}$ of SAM (Protocol 7b), weak signals (marked with circles) with an Rf close to the standard of trimethylresveratrol were observed, which suggested the occurrence of the methylation process. This observation correlates with a literature report by Couler et al. (1993). To confirm the results, an HPLC analysis was performed on the sample after the second day of biotransformation (Fig. 5). In addition to the signal of resveratrol (16 min), two main products with a retention time of 10.4 and $24.77 \mathrm{~min}$, respectively, were recorded. The product with the retention time at 24.77 min was similar to the tri- $O$-methyl derivative of resveratrol. This assumption was made due to the very small difference in theretention times of the discussed derivative $(24.77 \mathrm{~min})$ and the trimethylresveratrol standard (24.13 min) - Figure 6. Biotransformation carried out on a semi-preparative scale according to Protocol $7 \mathrm{~b}$ (for 6 days) resulted in the formation of the product with the retention time of $10.4 \mathrm{~min}$. This compound was isolated and identified as 3,5-dihydroxybenzaldehyde, which clearly confirmed the degradation of one of the rings of resveratrol as a result of the previous oxygenase activity (due to the action of resveratrol cleavage oxygenase 1 produced by Ustilago maydis; Brefort et al., 2011) towards the interphenyl $\mathrm{C} \alpha-\mathrm{C} \beta$ double bond in resveratrol (Mei et al., 2015), followed by the degradation of the 4-hydroxybenzyl ring. Unfortunately, much more interesting derivatives (Fig. 4, marked with circles- samples D1 and D2) which occurred on the first and the second day of these experiments (retention time $24.77 \mathrm{~min}$ ) were not obtained in sufficient quantities to carry out the structural analyses.

The next set of experiments (Protocol 7d) included the addition of two additives: $L$-methionine and SAM. $L$-methionine was added to improve the regeneration of SAM and to facilitate the methylation of resveratrol. As shown in Figure 3, as a result of the introduction of both the additives (SAM and $L$-methionine) to the biotransformation medium at the same time, a number of products were obtained, but most of them by the degradation processes. After the addition of $L$-methionine, the mycelium turned green, and it could not be excluded that methionine stimulated the fungal cells to synthesize colored secondary metabolites.

The performed experiments were not successful in terms of the goal of the study which was to achieve the biosynthesis of the methylated resveratrol derivatives using P. chrysosporium. However, the results of the studies are valuable as they fill, at least partially, the gap in the knowledge about the changes occurring in the physiology of a white-rot fungal strain under nonphysiological conditions.

\section{Conclusion}

Unfortunately, the biotransformation conditions suitable for obtaining the tri- $O$-methyl derivative of resveratrol were not identified from the conducted experiments. The major problem in using $P$. chrysosporium for the biomethylation of resveratrol was the difficulty in obtaining an adequate level of methyltransferase activity. This is mainly due to the poor understanding of the regulatory mechanisms of $P$. chrysosporium occurring in response to the different nutrient sources in the culture medium and the sensitivity of the species to temperature, light, shaking, and so on. However, the study results widened the knowledge of the physiology of this species.

\section{Acknowledgment}

This project was supported by the Wroclaw Centre of Bio- 
technology, under the Leading National Research Centre Programme (KNOW) for years 2014-2018 (Project 367/2016/ KNOW).

\section{References}

Adom K., Sorrells M., Liu R. (2003) Phytochemical profiles and antioxidant activity of wheat varieties. J. Agric. Food Chem. 51: 7825-7834.

Barluenga J., Andina F., Aznar F. (2006) Unprecedented reactivity pattern of chromium fischer carbene complexes. Direct application to one-pot synthesis of 4-aryl-3,4-dihydrocoumarins on a multigram scale. Org. Lett. 8: 2703-2706.

Baur J.A., Sinclair D.A. (2006) Therapeutic potential of resveratrol: the in vivo evidence. Nat. Rev. Drug. Discov. 5: 493-506.

Botella L., Najera C. (2004) Synthesis of methylated resveratrol and analogues by Heck reactions in organic and aqueous solvents. Tetrahedron 60: 5563-5570.

Brefort T., Scherzinger D., Limon M.C. et al (2011) Cleavage of resveratrol in fungi: characterization of the enzyme Rco1 from Ustilago maydis. Fungal Genet. Biol. 48: 132-143.

Catalgol B., Batirel S., Taga Y., Ozer N.K. (2012) Resveratrol: French paradox revisited. Front. Pharmacol. 3 (141): 1-18.

Chagas E.P., Durrant L.R. (2001) Decolorization of azo dayes by Phaneroachete chrysosporium and Pleurotus sajorcaju. Enzyme. Microb. Technol. 29: 473-477.

Chen C.L., Chang H.M., Kirk T.K. (1982). Aromatic acids produced during degradation of lignin in spruce wood by Phanerochaete chrysosporium. Holzforschung. 36: 3-9.

Coque J.J.R., Alvarez-Rodriguez M.L., Larriba G. (2003) Characterization of an inducible chlorophenol O-methyltransferase from Trichoderma longibrachiatum involved in the formation of chloroanisoles and determination of its role in Cork Taint of wines. Appl. Environ. Microbial. 69: 5089-5095.

Coulter C., Kennedy J.T., McRoberts W.C., Harper D.B. (1993) Purification and properties of an S-adenosylmethionine 2,4disubstituted phenol 0-methyltransferase from Phanerochaete chrysosporium. Appl. Environ. Microbiol. 59: 706-711.

de Carvalho C.C.C.R. (2017) Whole cell biocatalysts: essential workers from Nature to the industry. Microbial Biotechnol. 10: 250-263.

Demain A.L. (2000). Microbial buitechnology. Trends Biotechnol. 52: 455-463.

Dipak K., Maulik N. (2006) Resveratrol: A Preconditioning Agent. Mol. Interventions. 1(6): 36-47.

Dosoretez C.G., Chen A.H.C., Grethlein H.E. (1990) Effect of oxygenation conditions on submerged cultures of Phanerochaete chrysosporium. Appl. Microbiol. Biot. 34: 131-137.

Eriksson K.E., Gupta K., Nishida A., Rao R. (1984) Syringic acid metabolism by some white rot, soft rot and brown rot fungi. J. Gen. Microbiol. 130: 2457-2464.

Fillion E., Dumas A.M., Kuropatwa B.A., Malhotra N.R., Sitler T.C. (2006) $\mathrm{Yb}(\mathrm{OTf})_{3}$-catalyzed reactions of 5-alkylidene meldrum's acids with phenols: one-pot assembly of 3,4- dihydrocoumarins, 4-chromanones, coumarins, and chromones. J. Org. Chem. 71: 409-412.

Gallardo C., Jimenez L., Garcia-Conesa M.T. (2006) Hydroxycinnamic acid composition and in vitro antioxidant activity of selected grain fractions. Food Chem. 99: 455-463.

Gao D., Du L., Yang J., Wu W. \& Liang H. (2010) A critical review of the application of white rot fungus to environmental pollution control. Crit. Rev. Biotechnol. 30: 70-77.

Horinouchi S. (2009) Combinatorial biosynthesis of plant medicinal polyketides by microorganisms. Curr. Opin. Chem. Biol. 13: 197-204.

Houille B., Papon N., Boudesocque L., Bourdeaud E. et al. (2014) Antifungal activity of resveratrol derivatives against Candida species. J. Nat. Prod. 77: 1658-1662.

Jayashree C., Janshi G., Yeom I.T, Kumar S.A., Banu J.R. (2014) Effect of low temperature thermo-chemical pretreatment of dairy waste activated sludge on the performance of microbial fuel cell. Int. J. Electrochem. Sci. 9: 5732-5742.

Jeandet P., Clement C., Courot E. (2014) Resveratrol production at large scale using plant cell suspensions. Eng. Life Sci. 14: 622-632.

Jeffers M.R., McRoberts W.C., Harper D.B. (1997) Identification of a phenolic 3-O-methyltransferase in the lignin-degrading fungus Phanerochaete chrysosporium. Microbiology 143: 1975-1981.

Kang S.Y., Lee J.K., Choi O., Kim C.Y., Jang J.H., Hwang B.Y., Hong Y.S. (2014) Biosynthesis of methylated resveratrol analogs through the construction of an artificial biosynthetic pathway in E. coli. BMC Biotechnol. 14: 67.

Kim D.J., Huh J.H., Yang Y.Y., Kang C.M., Lee J.H., Hyun C.G., Hong S.K., Suh J.W. (2003) Accomulation of S-adenosyl-L-methionine enhances production of actinorhodin but inhibits sporulation in Streptomyces lividans TK23. J. Bacteriol. 185: 592-600.

Kozyra K., Brzezińska-Rodak M., Klimek-Ochab M., Żymańczyk-Duda E. (2013) Biocatalyzed kinetic resolution of racemic mixtures of chiral $\alpha$ - aminophosphonic acids. J. Mol. Catal. B: Enzym. 91: 32-36.

Koutaniemi S., Toikka M.M., Karkonen A., Mustonen M., Lundell T., Simala L.K., Kilpelainen I.A., Teeri T.H. (2005) Characterization of basic p-cumaryl and coniferyl alcohol oxidizing peroxidases from a lignin - forming Picea abies supension culture. Plant Mol. Biol. 58: 141-157.

Kris-Etherton P.M., Hecker K.D., Bonanome A., Coval S.M., Binkoski A.E., Hilpert K.F., Griel A.E., Etherton T.D. (2002) Bioactive compounds in foods: their role in the prevention of cardiovascular disease and cancer. Am. J. Med. 113: 71-88.

Manna C., Ragione F.D., Cucciola V., Borriello A., D’Angelo S., Galletti P., Zappia V. (1999) Biological effects of hydroxytyrosol, a polyphenol from olive oil endowed with antioxidant activity. Adv. Exp. Med. Biol. 472: 115-130.

Mazue F., Colin D., Gobbo J., Wegner M., Rescifina A., Spatafora C., Fasseur D., Delmas D., Meunier P., Tringali C., Latruffeet N. (2010) Structural determinants of resveratrol 
for cell proliferation inhibition potency: Experimental and docking studies of new analogs. Eur. J. Med. Chem. 45: 2972-2980.

McGuire M.A., Shilcrat S.C., Sorenson E. (1999) For a coumarin-derived substrate for hydrogenation. Tetrahedron Lett. 40: 3293-3296.

Mei Y.Z., Liu R.X., Wang D.P., Wang X., Dai C.C. (2015) Biocatalysis and biotransformation of resveratrol in microorganisms. Biotechnol Lett. 37: 9-18.

Montedoro G., Servili M., Baldioli M., Miniati E. (1992) Simple and hydrolyzable phenolic compounds in virgin olive oil. 1. Their extraction, separation, and quantitative and semiquantitative evaluation by HPLC. J Agric. Food Chem. 40: 1571-1576.

Paszczyński A, Łobarzewski J. (1984) Modifications of peroxidase polymorphism in trametes versicolor fungus after short-time incubation under starvation conditions. Biochem. Physiol. Pflanz. 179: 749-757.

Rimando A.M., Pan Z., Polashock J.J., Dayan F.E., Mizuno C.S., Snook M.E., Liu C.J., Baerson S.R. (2012) In planta production of the highly potent resveratrol analogue pterostilbene via stilbene synthase and O-methyltransferase coexpression. Plant Biotechnol. J. 10: 269-283.

Roberti M., Pizzirani D., Simoni D., Rondanin R., Baruchello R., Bonora C., Buscemi F., Grimaudo S., Tolomeo M. (2003) Synthesis and biological evaluation of resveratrol and analogues as apoptosis-inducing agents. J. Med. Chem. 46: 3546-3554.

Solladie G., Pasturel-Jacope Y., Maignan J. (2003) A re-investigation of resveratrol synthesis by Perkins reaction. Application to the synthesis of aryl cinnamic acids. Tetrahedron. 59: 3315-3321.

Somerville G.A., Proctor R.A. (2013) Cultivation conditions and the diffusion of oxygen into culture media: The rationale for the flask-to-medium ratio in microbiology. BMC Microbiol. 13: 9-10.

Stervbo U., Vang O., Bonnesen Ch. (2007) A review of the content of the putative chemopreventive phytoalexin resveratrol in red wine. Food Chem. 2: 449-457.

Straathof A.J. (2013) Transformation of biomass into commodity chemicals using enzymes or cells. Chem. Rev. 114: 1871-1908.
Thanh Mai Pham L., Kim Y.H. (2016) Discovery and characterization of new $O$-methyltransferase from the genome of the lignin-degrading fungus Phanerochaete chrysosporium for enhanced lignin degradation. Enzyme. Microb. Technol. 82: 66-73.

Visioli F., Bellomo G., Galli C. (1998a) Free radical-scavenging properties of olive oil polyphenols. Biochem. Biophys. Res. Commun. 247: 60-64.

Visioli F., Galli C. (1998b) Olive oil phenols and their potential effects on human health. J. Agric. Food. Chem. 46: 4292-4296.

Wachtmeister J., Rother D. (2016) Recent advances in whole cell biocatalysis techniques bridging from investigative to industrial scale. Curr. Opin. Biotechnol. 42: 169-77.

Wang H., Dong Y., Xiu Z.L. (2008) Microwave-assisted aqueous two-phase extraction of piceid, resveratrol and emodin from Polygonum cuspidatum by ethanol/ammonium sulphate system. Biotechnol. Lett. 30: 2079-2084.

Wanga D.G., Liub W.Y., Chen G.T. (2013) $A$ simple method for the isolation and purification of resveratrol from Polygonum cuspidatum. J. Pharm. Anal. 3(4): 241-247.

Yu G., Wen X., Li R. \& Qian Y. (2006) In vitro degradation of a reactive azo dye by crude ligninolytic enzymes from no immersed liquid culture of Phanerochaete chrysosporium. Process. Biochem. 41: 1987-1993.

Yu J., Gaunt M.J., Spencer J. (2002) Convenient preparation of trans-arylalkenes via palladium(II)-catalyzed isomerization of cis-arylalkenes. J. Org. Chem. 67: 4627-4629.

Zhang K., Xu Y., Hua X., Han H., Wang J., Wang J., Liu Y., Liu Z. (2008) An intensified degradation of phenanthrene with macroporous alginate-lignin beads immobilized Phanerochaete chrysosporium. Biochem. Eng. J. 41: 251-257.

Zhuang X., Dong X., Ma S., Zhang T. (2008) Selective on-line extraction of transresveratrol and emodin from Polygonum cuspidatum using molecularly imprinted polymer. J. Chromatogr. Sci. 46: 739-742

Żymańczyk-Duda E., Brzezińska-Rodak M., Kozyra K., KlimekOchab M. (2015) Fungal platform for direct chiral phosphonic building blocks production. Closer look on conversion pathway. Appl. Biochem. Biotechnol. 175: 1403-1411. 